\title{
A POSITION-BASED EXPLICIT FORCE CONTROL STRATEGY BASED ON ONLINE TRAJECTORY PREDICTION
}

\author{
Xianjun Sheng, ${ }^{*}$ Lei Xu, ${ }^{*}$ and Zhou Wang*
}

\begin{abstract}
In the mirror milling process of large thin-walled parts, maintaining a constant supporting force is crucial for improving the workpiece local stiffness to suppress vibration. Aiming at the flexible characteristics of large thin-walled parts, this work presents a position-based force explicit control strategy based on online trajectory prediction. An online trajectory pre-modification force controller is proposed, it contains a position pre-modification module and a typical feedback controller for coarse adjustment and precise adjustment. The position pre-modification value is obtained by online trajectory prediction, and the prediction method relies on an online force and normal direction measurement system. The position premodification module can reduce the interference of the flexible characteristics on the force control. Numerical simulation and experiment are carried out, the control results show: the performance of dynamic force tracking method proposed is improved compared with the traditional position-based explicit control strategy with a typical feedback controller.
\end{abstract}

\section{Key Words}

Robot control; compliant motion strategy; trajectory prediction; position-based explicit force control

\section{Introduction}

In the aerospace manufacturing field, the manufacturing of large thin-walled parts is quite difficult because of the following characteristics: large area, complex shape, low structure stiffness and easy deformation. For a long time, chemical milling is widely used in the manufacturing of large thin-walled parts, but there are some inevitable problems such as chemical pollution, long production period and a lot of material waste. The mirror milling method has features such as high quality, high efficient and low power consumption. It has recently become a research focus in the manufacturing field of large thin-walled parts. In the mirror milling process of large thin-walled parts, low

* Institute of Electrical Engineering, Dalian University of Technology, Dalian, China; e-mail: sxianjun@mail.dlut.edu.cn, \{leixu,wangzhou\}@mail.dlut.edu.cn

Recommended by Dr. Francesco Pierri

(DOI: 10.2316/Journal.206.2017.1.206-4899) structure stiffness will lead to vibration. Normal direction constant supporting force and milling-supporting synchronization can improve the local rigidity so as to suppress vibration. The milling-supporting synchronization method has been discussed in [1]. This paper is focused on how to maintain a constant supporting force. Usually, industrial robots serve as the manipulators in the mirror milling system. Obviously, position control of the robots itself is not capable for the task, force sensing and compliance control is necessary as well.

Typical active compliance control of the robot is divided into impedance control, hybrid force/position control and explicit force control currently [2]-[4]. In the impedance control, the target impedance is set according to the environment information, so that the robot end can achieve the capability of compliant motion. Its control precision depends on the accurate knowledge of the environment [5], [6]. The basic idea of hybrid force/position control is to divide the end space of the robot into two orthogonal spaces by selection matrix. Position control and force control are applied on two spaces to achieve the capability of compliant motion [7]-[9]. Explicit force control involves the direct command and measurement of force values, and offers the possibility of controlling the contact force to a desired value [10], [11]. Komati in [12] presented a new position-based impedance control scheme to estimate environmental stiffness according to the past value of the position measurement. Moreover, there are some other papers focused on estimating the stiffness of environment on line [13], [14]. However, the precondition of these methods is that the stiffness is invariant with time. When the stiffness is unpredicted and changed in real time, whether these methods proposed by previous passages are effective is not convinced. The hybrid force/position control strategy needs to divide the constraint frame into position-controlled and force-controlled subspaces, and the subspaces vary with the supporting surface normal direction varying. So, it will add more complexity to the controller and it may decrease the stability of the system. The explicit force control method has stronger robustness and stability. Compared with an impedance control strategy and hybrid force/position control strategy, an explicit force control strategy is more suitable for the task. Two types 
of explicit force control strategy exist: force-based explicit force control strategy and position-based explicit force control strategy [15]. Industrial robots commonly adopt a joint position control scheme. So, it is difficult to apply the force-based explicit force control strategy. The position-based explicit force control strategy which corrects expected trajectory by the feedback force information is relatively easily implemented in industrial applications [16], [17].

The position-based explicit force control strategy has been widely developed in the field of scientific research and engineering applications such as robot grasping, automatic assembly and precision polishing. Liu [18] implemented the position-based explicit force control strategy to control the milling force of a rail milling system. An internal model controller is applied as the force controller. The simulation results show that the milling force can be tracked with zero steady-state error under this control method. Sun [19] implemented the position-based explicit force control architecture to an automatic robotic polishing system, which is aimed at the polishing of titanium alloy curved parts. An anti-saturation integral separated fuzzy proportional-integral (PI) controller is implemented as the force controller. The controller can imitate the manual polishing operation and prevent undesirable vibrations and mechanical collisions. The experimental result shows that the proposed method has a perfect control effect on the contact force. Ahn [20] applied the position-based explicit force control technique to robot arms for a mobile robot. A proportional-integral-derivative (PID) controller is applied as the force controller. Experimental studies of force control applications that robot arms interact with a human operator are conducted, and it shows that the robot arm is well regulated to follow the desired force. Perez Plius [21] combined the position-based explicit force control scheme and admittance control scheme to robot arm interaction with environment. When approaching to the environment, the admittance control scheme is applied. When contacting with the environment, the explicit force control scheme is used and an integral controller is applied as the force controller. Fuzzy logic scheduling is used to switch between the two control schemes. The experimental result shows that the proposed method is a good candidate for industrial applications. The research studies above applied the position-based explicit force control strategy to different applications, and different feedback controllers are chosen as the force controllers. But they are all aimed at works that the environment is not or slowly deformed. In these works, a feedback force controller is reasonable and it can track the desired force precisely. In the mirror milling of large thin-walled parts however, there is unpredictable and rapid deformation in the machining process due to the weak-rigidity characteristics of the structural parts, so the dynamic force tracking performance of a feedback force controller will decrease significantly. A pre-modification of the supporting point is an effective supplement for a traditional feedback force controller, as the pre-modification can improve the response speed of the force controller.

In this paper, an online trajectory pre-modification (OTP) force controller is applied to the position-based

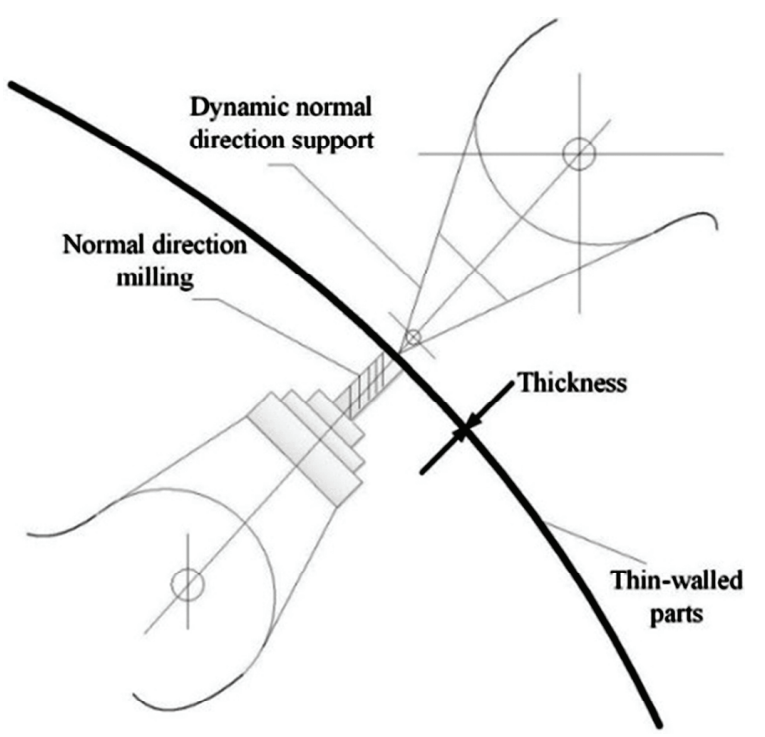

Figure 1. Mirror milling schematic diagram for large thinwalled parts.

explicit force control strategy. In the OTP force controller, a position pre-modification module is added to a feedback controller and the pre-modification value is obtained by an online trajectory prediction method which relies on an online measurement system. The force controller proposed can provide an extra coarse adjustment compared with a typical feedback controller, so it can improve the dynamic force tracking performance compared with the traditional position-based explicit force control strategy with a feedback force controller.

\section{System Composition}

Typical mirror milling schematic diagram for large thinwalled parts is shown in Fig. 1. In a typical mirror milling system, the cutting tool and the supporting head are located at two sides of the large thin-walled parts. The supporting point needs to keep mirror symmetry with the milling point, and the supporting force needs to be constant. The requirements above can improve the milling parts' local rigidity which is vital for vibration suppression.

In the mirror milling system, a high rigidity manipulator is the requirement for maintaining enough local rigidity of the milling parts, so it is also crucial for vibration suppression. A tricept hybrid mechanism consists of a 3-DOF paralleling mechanism and a $2-\mathrm{DOF}$ rotating head. It is chosen as the actuator considering its big stiffness as well as its relatively large operating range, which is necessary for large thin-walled parts machining too. In the supporting side, so as to achieve normal direction constant force supporting, a supporting bar with measurement function is mounted at the end of the hybrid mechanism. The measurement module consists of an eddy current sensor array and a piezoelectric strain force sensor. They are mounted on the supporting bar to measure the normal direction and the supporting force, respectively. The eddy current sensor array consists of three eddy current sensors. The normal 


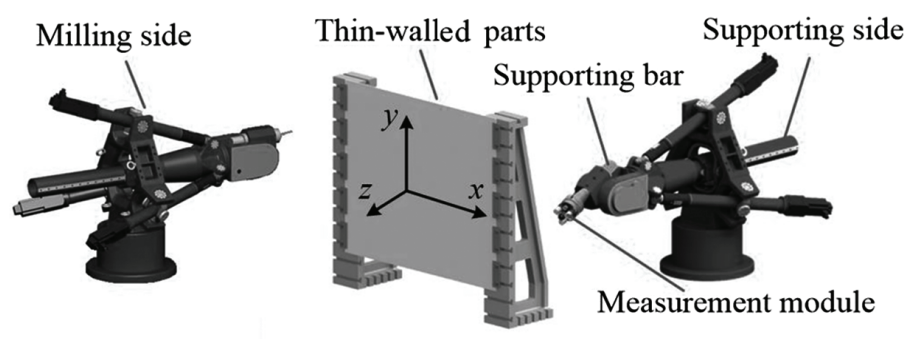

(a)

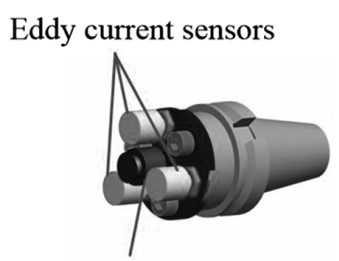

Force sensor

(b)

Figure 2. Diagram of mirror milling system: (a) entire configuration and (b) measurement module.

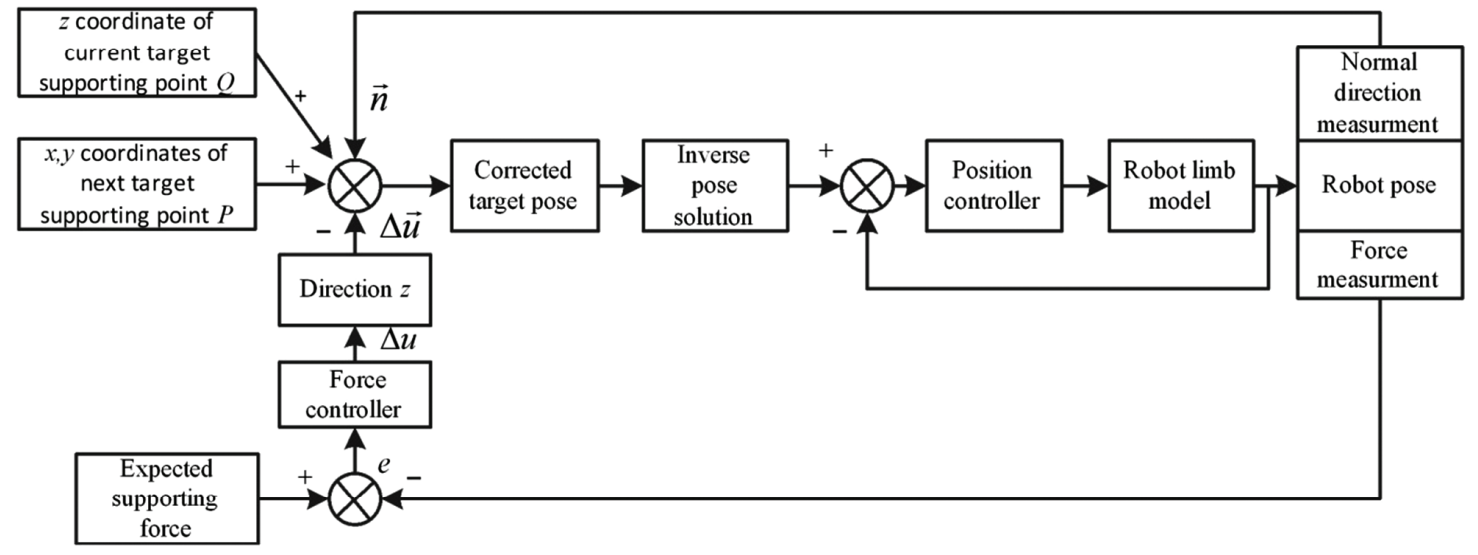

Figure 3. Traditional position-based explicit force control strategy.

direction of the supporting surface local can be calculated by the distances which are measured by the three eddy current sensors. The measurement system is also the basis of online trajectory prediction and pre-modification. The systematic configuration diagram of the mirror milling system is shown in Fig. 2.

\section{Force Control Strategy}

The traditional position-based explicit force control scheme is shown in Fig. 3.

According to the characteristics that the local of large thin-walled parts is similar to a plane, as is shown in Fig. 2, the initial normal direction of the plane is defined as the $z$ axis. In the traditional position-based explicit force control strategy, as is shown in Fig. 3, the $x, y$ coordinates of the target supporting point trajectory are given in advance according to the trajectory planning. The $z$-axis of the target supporting point is adjusted by the force controller, so as to track the constant supporting force along the supporting bar.

\subsection{Position-based Explicit Force Control Strategy with Online Trajectory Prediction Method}

The difference between the traditional position-based explicit control strategy mentioned in Fig. 3 and the proposed method lies in the force controller. Usually, a feedback force controller is applied in the traditional position-based explicit control strategy. In the proposed method, an OTP force controller is proposed aimed at the easy deformation characteristics of the structural parts. The schematic of the position-based explicit force control strategy with an OTP force controller is shown in Fig. 4.

In the OTP force controller, there are an antisaturation integral separated PI controller and a position pre-modification module based on online trajectory prediction. In the anti-saturation integral separated PI controller, when the error between the actual supporting force and expected supporting force is large, a proportional control method is used to significantly reduce the steady-state errors and make the system response fast. When the error is small, another PI control method is used to eliminate the static error and improve the adaptability of the system on the environment. Moreover, an anti-saturation unit is used to limit the range of output value to prevent undesirable vibrations and mechanical collisions. The extra position pre-modification module is specially designed for the easy deformation characteristics of the large thin-walled parts, and it can reduce the interference on the force control caused by the characteristics. The anti-saturation integral separated PI controller serves as a precise adjustment, the position pre-modification module based on online trajectory prediction serves as a coarse adjustment. So, the OTP force controller has a faster response speed and can obtain a better force tracking performance. 


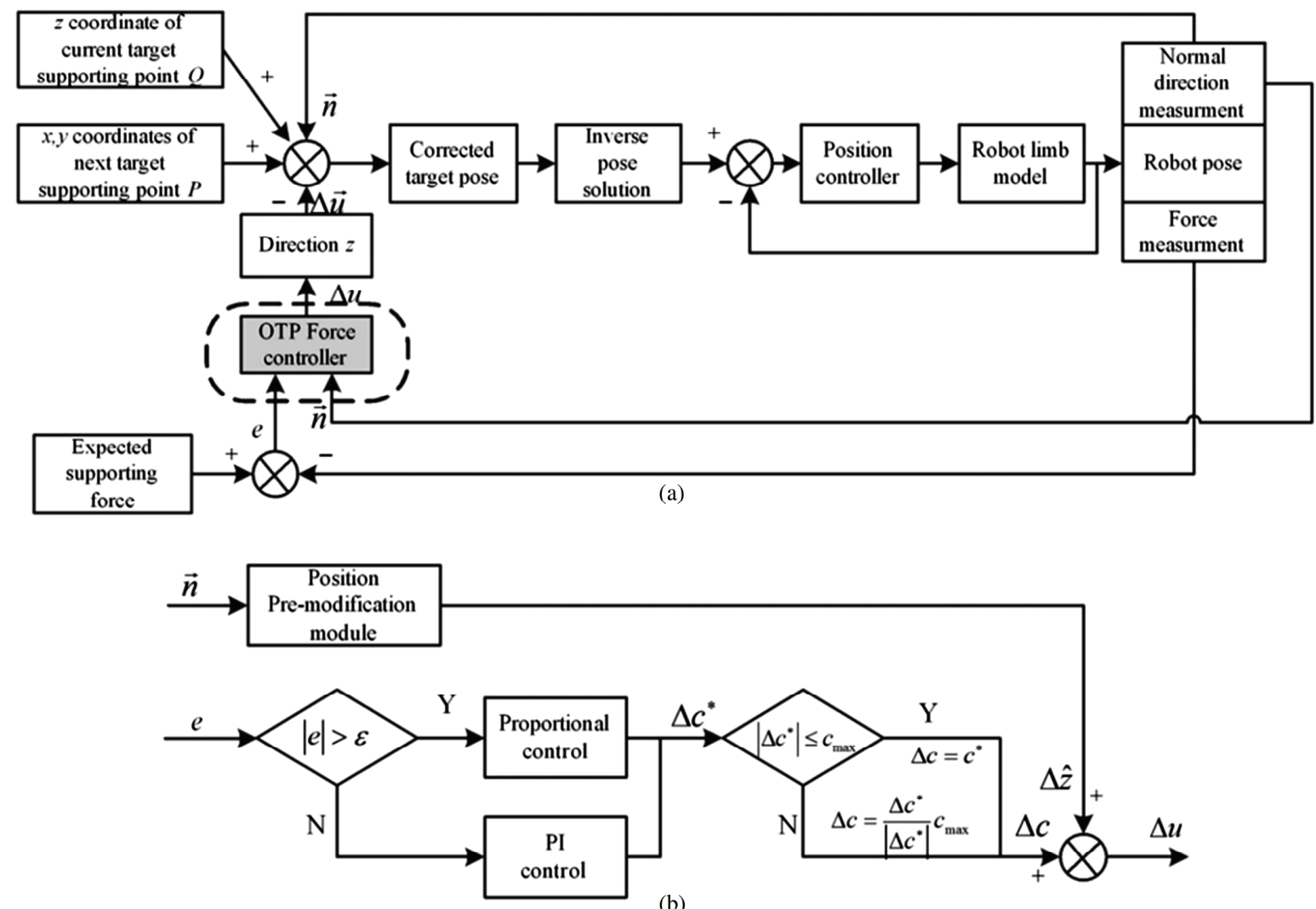

(b)

Figure 4. Proposed position-based explicit force control strategy: (a) control block and (b) OTP controller.

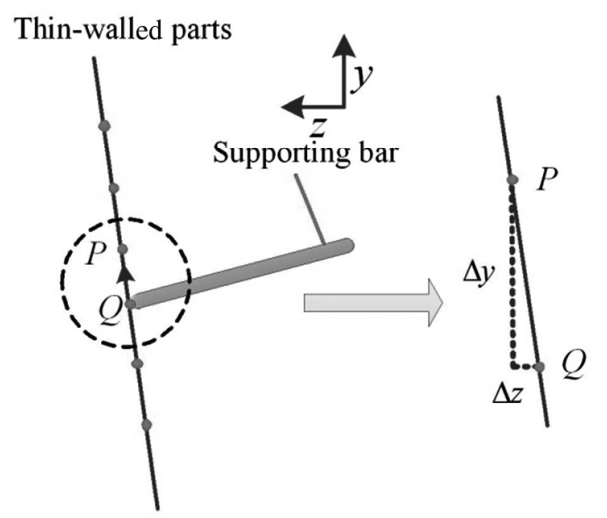

Figure 5. Schematic diagram of online trajectory prediction.

\subsection{Online Trajectory Prediction Method}

The position pre-modification module relies on online position prediction of the supporting position. The position of supporting point is reflected in Fig. 5. $Q$ denotes the current target supporting point and $P$ denotes the next target supporting point. The distances between $Q$ and $P$ in the $x$ - and $y$-axis are decided by the trajectory planning. The distance between $Q$ and $P$ in the $z$-axis is predicted by the distances between $Q$ and $P$ in the $x$ - and $y$-axis and the normal direction of $Q$.

The supporting point local can be simplified as a plane as the curvature of large thin-walled parts is small enough. In addition, the normal direction variation and the deformation during the machining process from $Q$ to $P$ can be neglected as the distance is small enough. Set the normal direction of the current supporting point measured by eddy current sensor array as a vector $\vec{n}=\left(\begin{array}{lll}x_{n} & y_{n} & z_{n}\end{array}\right)$, and may express the vector from $Q$ to $P$ as $\overrightarrow{Q P}=\left(\begin{array}{lll}\Delta x & \Delta y & \Delta z\end{array}\right)$. As the supporting point local is shown as a plane, which means $\overrightarrow{Q P} \cdot \vec{n}=0$, the correction of predicted position in the $z$-axis between $Q$ and $P$ can be expressed as follows:

$$
\Delta \hat{z}=\frac{x_{n} \Delta x+y_{n} \Delta y}{-z_{n}}
$$

So, the position pre-modification value in the OTP force controller is $\Delta \hat{z}=\left(x_{n} \Delta x+y_{n} \Delta y\right) /-z_{n}$.

\section{Simulation and Experiment Study}

\subsection{Control Strategy Simulation}

To verify the performance of the force tracking applied position-based explicit force control strategy with an OTP force controller, numerical simulation is implemented. A tricept hybrid manipulator model is established in SimMechanics according to the manipulator actual parameters, as is shown in Fig. 6(a). The limb servo motors are controlled based on the three closed-loop PID control scheme, which is commonly used in industry. The limb motor control model is established in Simulink, as is shown in Fig. 6(b). 


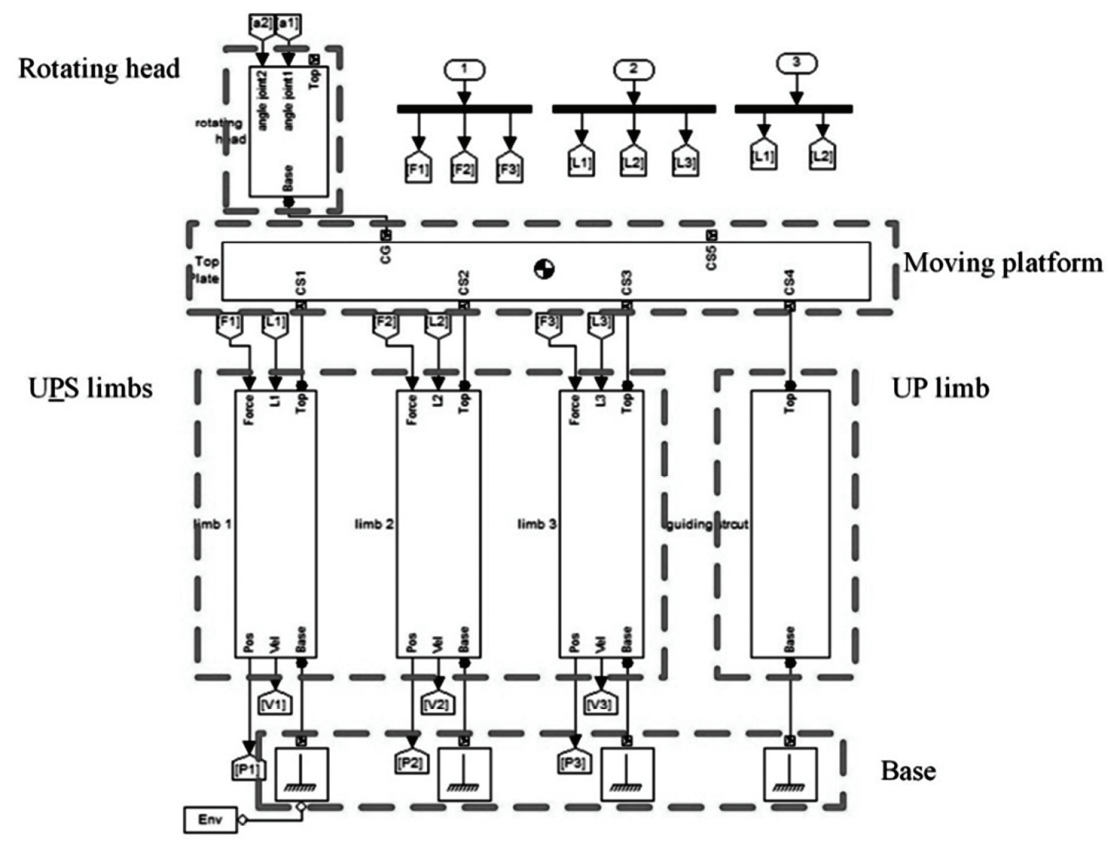

(a)

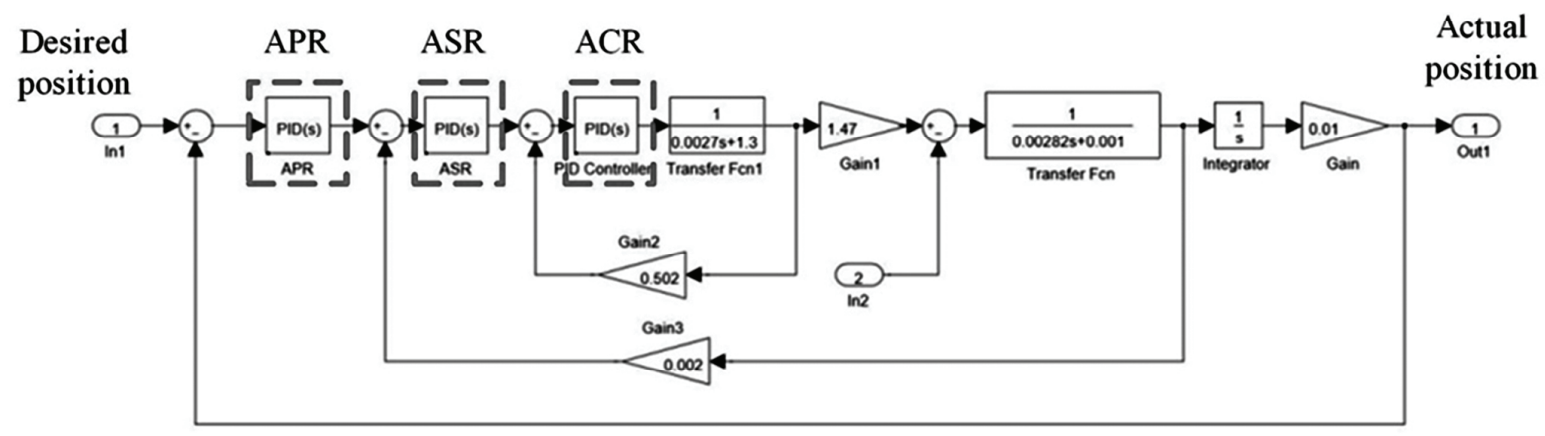

(b)

Figure 6. Fundamental models of the system: (a) tricept manipulator model and (b) limb motor model.

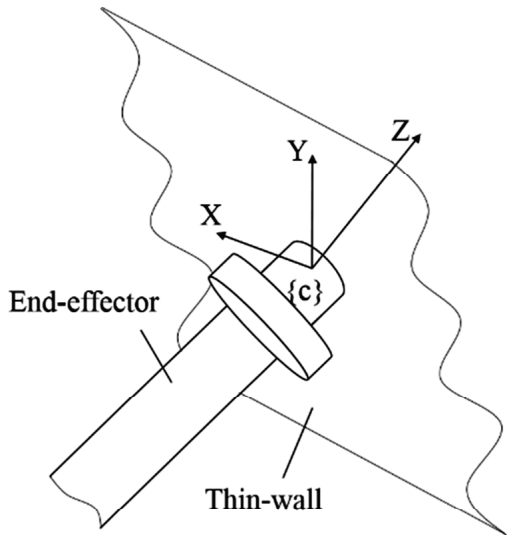

Figure 7. The sketch map of supporting simulation.

Due to the characteristics that the local structure of large thin-walled parts is approximate to a plane. As is shown in Fig. 7, two small amplitude sine signals with different frequencies are added together to represent the deformation of supporting surface along the $y$-axis direction. The bodily movement of the supporting surface is a sine signal along the $z$-axis direction.
The movement of the supporting manipulator is divided into two phases: adjustment phase and dynamic tracking phase. In the adjustment phase $(0-2 \mathrm{~s})$, it moves along the surface normal direction, $z$-axis direction, to achieve the desired constant supporting force. In the dynamic tracking phase $(2-6 \mathrm{~s})$, it needs maintaining the desired constant supporting force while tracking the target supporting trajectory in the $x$ - and $y$-axis. The desired force along the supporting bar is set as $20 \mathrm{~N}$ in both phases. The position-based explicit force control strategy with an OTP force controller and the traditional position-based explicit force control strategy with a typical PI force controller (anti-saturation integral separated PI controller) are programmed in Simulink. The simulation results are shown in Figs. 8 and 9.

In the adjustment phase, the position pre-modification module in the OTP force controller does not work. It results that the performance of OTP controller is the same as the typical PI controller in the adjustment phase. Therefore, the force tracking performance of the two control methods is the same as shown in Fig. 8, for the same anti-saturation integral separated PI force controller is applied. As is shown in Fig. 8(b), for the two methods, the responses are the same and the 


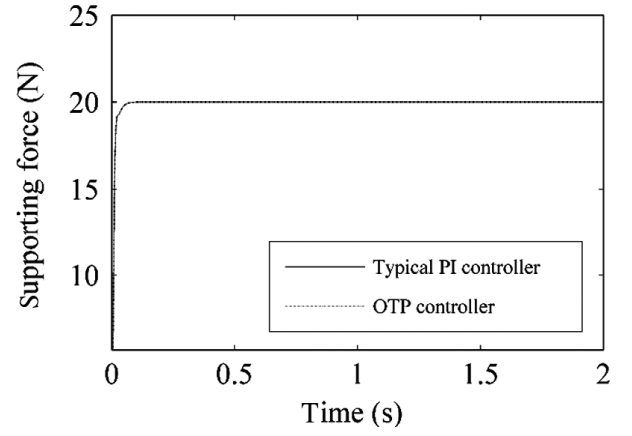

(a)

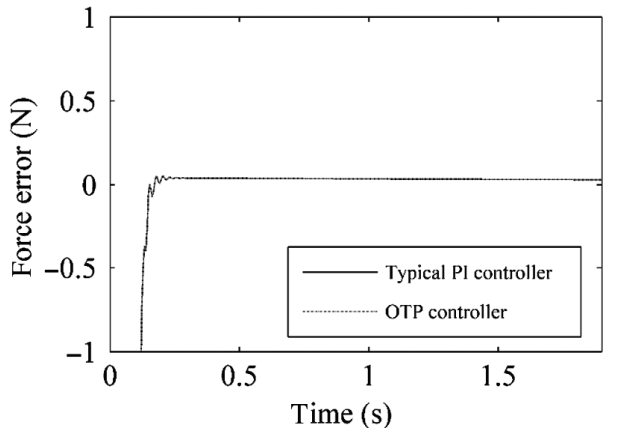

(b)

Figure 8. Simulated performance in adjustment phase: (a) desired force $20 \mathrm{~N}$ in overall view and (b) force error.

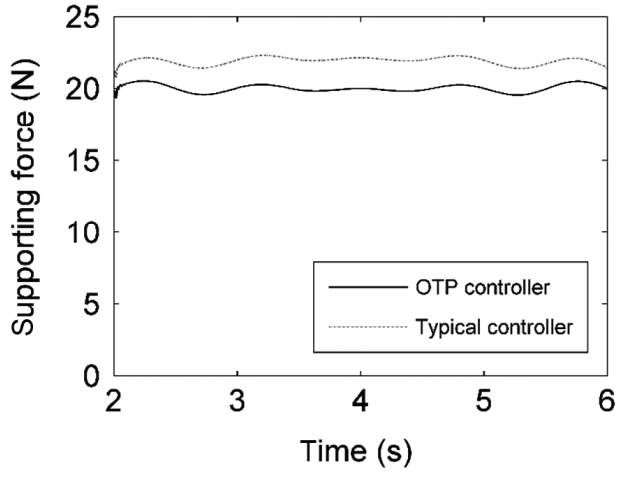

(a)

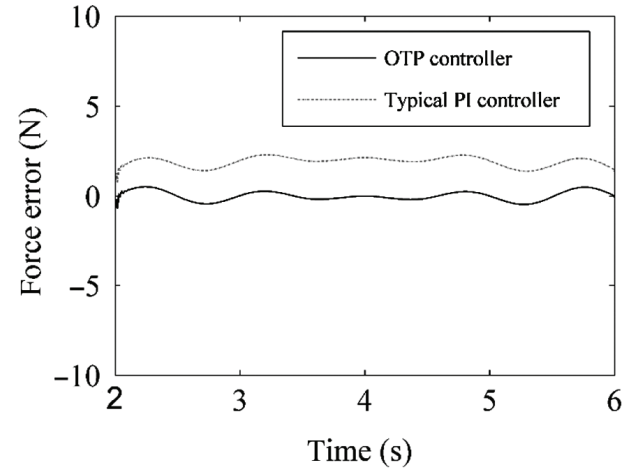

(b)

Figure 9. Simulated performance in dynamical tracking phase: (a) desired force $20 \mathrm{~N}$ in overall view and (b) force error.

steady-state force error is less than $0.2 \mathrm{~N}$. In the dynamic tracking phase, compared with an OTP controller, the typical PI controller exhibits an offset error caused by the designed deformation of environment in the simulation. In the dynamic tracking phase, the online trajectory prediction module can detect the environmental deformation and then effectively reduce the offset error caused by the deformation of thin-walled. As is shown in Fig. 9, when the position-based explicit force control strategy with an OTP force controller is applied, the maximum force tracking error is less than $1 \mathrm{~N}$. Compared with the traditional position-based explicit force control strategy with a typical PI force controller, the proposed method can significantly improve the dynamic force tracking performance, and the maximum force tracking error is cut down from 2.5 to $1 \mathrm{~N}$ as is shown in Fig. 9(b). In Fig. 9, there are a lot of oscillations which is due to the setting of supporting surface and the limb motor model. The force tracking on the limitation of motor response speed lags behind the deformation of supporting surface. It results in unavoidable oscillations.

\subsection{Experiments}

To verify the actual performance of the proposed force control method, experiments are taken based on a simplified supporting system. The system consists of motion module and measurement module. The motion module of the system consists of a cross worktable actuator, servo motor and driver, motion control card and PC. The positioning precision of the motion module is $2 \mu \mathrm{m}$. The measurement module is previously introduced in Section 2. It consists

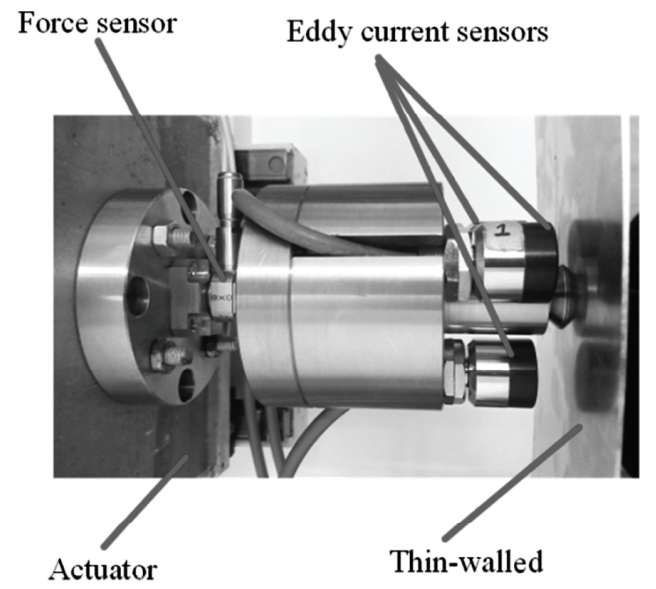

Figure 10. The supporting force control experiment.

of three eddy current sensors (KEYENCE EX-422 V), a force sensor (KISTLER 9317C) and a data collecting card. Instead of tracking the normal direction of the supporting point which is required in the mirror milling process, the supporting bar is fixed in the direction of $z$-axis as the direction cannot be changed based on the simplified supporting system due to lack of DOF. But it does not affect proving the effectiveness of the control algorithm. The force controller is programmed by LabVIEW in PC and position command outputted by the force controller is sent to the motion control card. The position control is completed by the motion control card. The large thin-walled parts are simulated by a $400 \mathrm{~mm} \times 100 \mathrm{~mm} \times 1 \mathrm{~mm}$ aluminium plate. The experiment is set as shown in Fig. 10. 


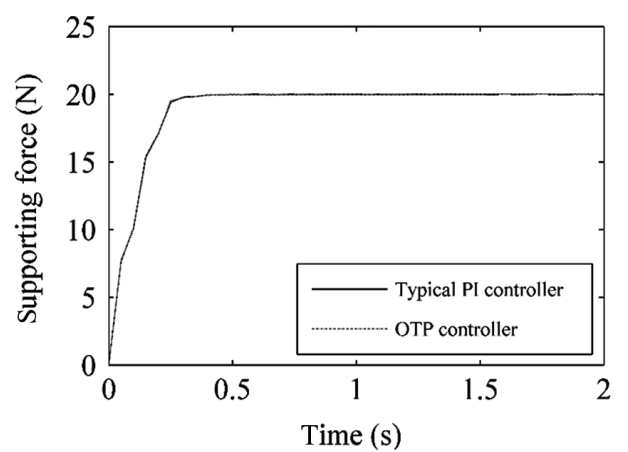

(a)

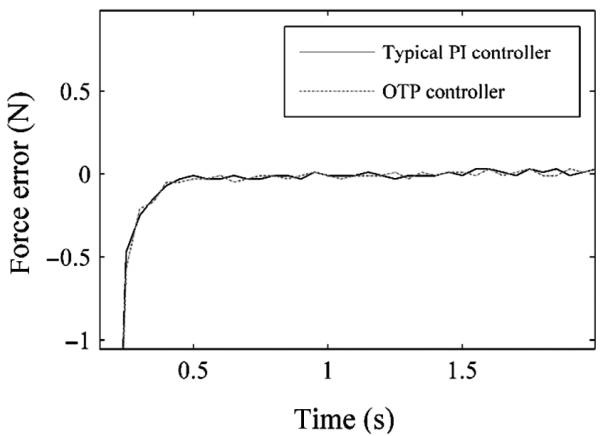

(b)

Figure 11. Experimental performance in adjustment phase: (a) desired force $20 \mathrm{~N}$ in overall view and (b) force error.

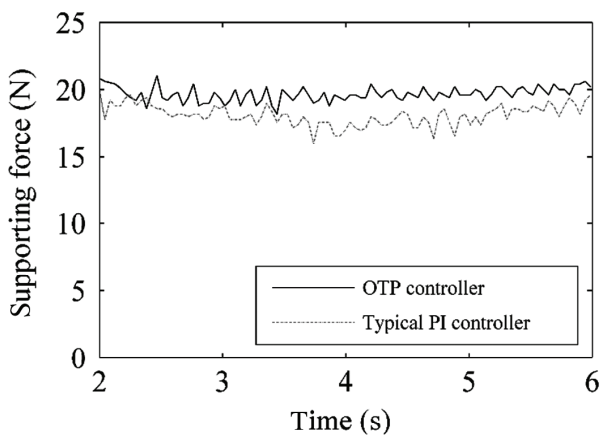

(a)

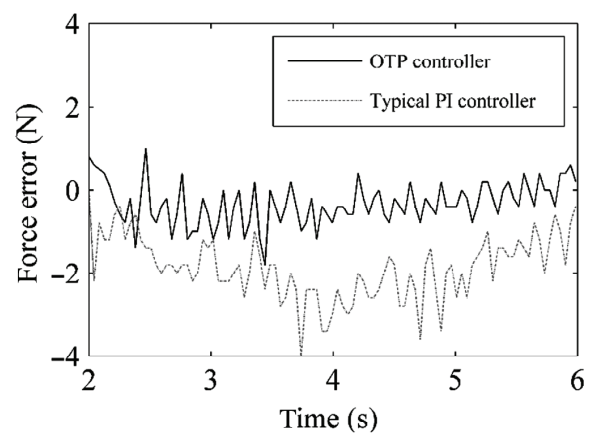

(b)

Figure 12. Experimental performance in dynamical tracking phase: (a) desired supporting force $20 \mathrm{~N}$ in overall view and (b) force error.

The position-based explicit force control strategy with an OTP force controller and the traditional positionbased explicit force control strategy with a typical PI force controller are applied, the desired force along the supporting bar is set as $20 \mathrm{~N}$. The same as the simulation, the movement is divided into two phases: adjustment phase and dynamic tracking phase. The experiemntal results of the two control methods are shown in Figs. 11 and 12 .

As is shown in Fig. 11, in the adjustment phase, the force tracking performance of the two control methods are similar, the steady-state force errors are both less than $0.2 \mathrm{~N}$. As is shown in Fig. 12, in the dynamic tracking phase, the proposed position-based explicit force control strategy with an OTP force controller can significantly improve the dynamic force tracking performance. The maximum force tracking error cut down from 4 to $1.5 \mathrm{~N}$, and the root mean square value of force error reduces from 2 to $0.6 \mathrm{~N}$. Compared with Fig. 11, there are a lot of oscillations in Fig. 12. On account of the oscillations that are caused by the surface irregularities of the large thinwalled, these oscillations are hard to reduce. When the supporting bar following with the target supporting trajectory, the force sensor is sensitive to the force change caused by the supporting bar encountering a bump on the surface. The experiment results demonstrate that the OTP control scheme can improve the dynamic force tracking performance significantly.

\section{Conclusion}

This paper proposes a position-based explicit force control method based on online trajectory prediction, dedicating to the supporting force control of a large thin-walled parts mirror milling process. Different from the traditional position-based explicit force control method which implements a typical feedback force controller, an OTP force controller is proposed, aiming at the easy deformation characteristics of large thin-walled parts. The OTP force controller adds a position pre-modification module based on the online trajectory prediction method to a feedback controller. So, it can reduce the influence of the workpiece deformation on the force control. The simulation and experiment results show that the proposed method can improve the dynamic force tracking performance significantly in the mirror milling of large thin-walled parts. The reported methodology can be extended for the force control in other tasks that the environment is easily deformed.

\section{Acknowledgement}

The research is supported by the "973" National Program (Grant No. 2014CB046603). 


\section{References}

[1] S. Xianjun, Z. Wang, L. Xu, et al., Coordinated motion strategy for multi-manipulators system based on joint space synchronized cross-coupled control, in H. Liu, N. Kubota, X. Zhu, R. Dillmann, and D. Zhou (eds.), Intelligent robotics and applications (Portsmouth UK: Springer, 2015), 396-407.

[2] S.K. Singh and D.O. Popa, An analysis of some fundamental problems in adaptive control of force and impedance behavior: theory and experiments, IEEE Transactions on Robotics 8 Automation, 11(6), 1996, 912-921.

[3] M. Homayounzade and M. Keshmiri, Adaptive position/force control of robot manipulators with force estimation, 2014 Second RSI/ISM Int. Conf. IEEE Robotics and Mechatronics (ICRoM), Tehran, Iran, 2014, 736-741.

[4] Q. Xu, New robust position and force regulation for a compliant microgripper, 2013 IEEE Int. Conf. IEEE Automation Science and Engineering (CASE), Guangzhou, China, 2013, 801-806.

[5] N. Hogan, Impedance control: An approach to manipulation, ASME Journal of Dynamic Systems, Measurement and Control, 107(1) 1985, 304-313.

[6] Z. Jiang, et al., Novel explicit force control strategy based on impedance inner control, Acta Aeronautica et Astronautica Sinica, 30(8), 2009, 1515-1520.

[7] M.H. LARaibert and J.J. Craig, Hybrid position/force control of manipulators, Journal of Dynamic Systems Measurement $\&$ Control, 103(2), 1980, 126-133.

[8] H. Bolandi, et al., Position/force control of a dual cooperative manipulator system based on a singularly perturbed dynamic model, International Journal of Robotics $\& 3$ Automation, 27(1), 2012, 76-91.

[9] A. Rodriguez-Angeles, M.A. Arteaga-Perez, R.D.J. PortilloVelez, and C.A. Cruz-Villar, Transparent bilateral masterslave control based on virtual surfaces: stability analysis and experimental results, International Journal of Robotics 83 Automation, 30(2), 2015, 128-139.

[10] H. Seraji, Adaptive admittance control: an approach to explicit force control in compliant motion, 1994 IEEE Int. Conf. IEEE Robotics and Automation, Stockholm, Sweden, 1994, 2705-2712.

[11] B. Siciliano and L. Villani, Robot force control, Kluwer Academic Publishers International Series in Engineering and Computer Science, The Springer International Series in Engineering and Computer Science, 540, 1999.

[12] B. Komati, C. Clevy, and P. Lutz, Force tracking impedance control with unknown environment at the microscale, 2014 IEEE International Conference on IEEE Robotics and Automation (ICRA), Hong Kong, China, 2014, 5203-5208.

[13] J. Li, L. Liu, Y. Wang, and W. Liang, Adaptive hybrid impedance control of robot manipulators with robustness against environment's uncertainties, Proceedings of 2015 IEEE International Conference on Mechatronics and Automation, 2015 August 2-5, Beijing, China, 1846-1851.

[14] J. Seul, T. C. Hsia, and R.G. Bonitz, Force tracking impedance control of robot manipulators under unknown environment, IEEE Transactions on Control Systems Technology, 12(3), 2004, 126-133.

[15] R. Volpe and P. Khosla, A theoretical and experimental investigation of explicit force control strategies for manipulators, IEEE Transactions on Automatic Control, 38(11), 1993, $1634-1650$

[16] T. Winiarski and A. Woźniak, Indirect force control development procedure, Robotica, 31(3), 2013, 465-478.

[17] B. Komati, et al., Explicit force control vs impedance control for micromanipulation, ASME 2013 International Design Engineering Technical Conferences and Computers and Information in Engineering Conference American Society of Mechanical Engineers, Las Vegas, America, 2013.

[18] Z.B. Liu, Research on the internal model control of the constant milling force of the rail milling train, Journal of Railway Science \& Engineering, 57(4) 2013, 117-122.

[19] H. Du, Y. Sun, et al., Automatic robotic polishing on titanium alloy parts with compliant force/position control, Proceedings of the Institution of Mechanical Engineers Part B Journal of Engineering Manufacture, 229(7), 2015, 217-224.
[20] J.K. Ahn, S. Jung, and J.K. Ahn, Position-based force control application of a mobile robot with two arms, Journal of Institute of Control, 19(4), 2013, 316-321.

[21] M. Perez Plius, et al., Fuzzy controller scheduling for robotic manipulator force control, 2012 12th IEEE Int. Workshop on IEEE Advanced Motion Control (AMC), Auckland, The New Zealand, 2012, 1-8.

\section{Biographies}

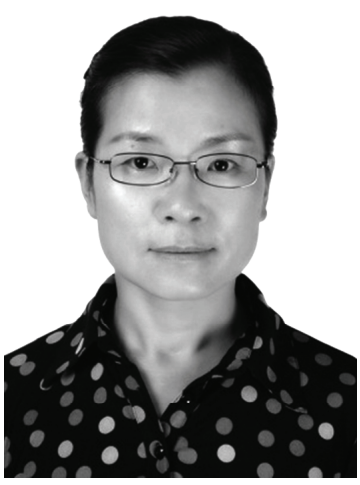

Xianjun Sheng was born in 1969. She received her B.E. degree in measuring and testing technologies and instruments and Ph.D. degree in mechanical manufacture and automation from Dalian University of Technology, Dalian, China, in 1997 and 2003, respectively. She is currently a Professor with School of Electrical Engineering in Dalian University of Technology. Her current research interests include digital manufacturing technology, simulation and calculation for microwave components.

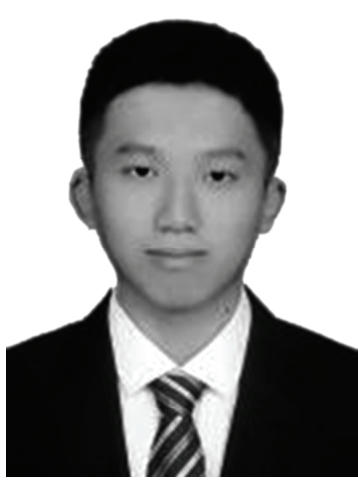

Lei $X u$ was born in 1990 . He is currently a power electronics and power transmission Master of Dalian University of Technology. He received his Bachelor's degree from Dalian University of Technology, Dalia, China, in 2013. His research activities deal with the robotic control, artificial intelligence and compliant control.

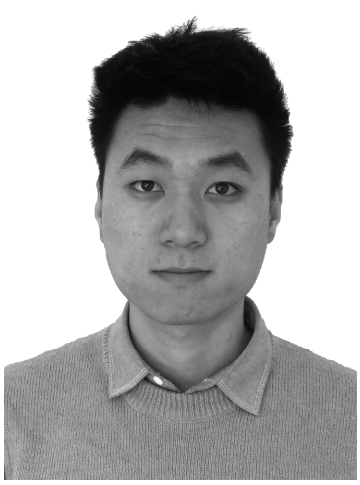

Zhou Wang is a power electronics and power transmission Master of Dalian University of Technology. He received his Bachelor's degree from Dalian University of Technology, Dalia, China, in 2013. His research activities deal with the robotic control, synchronization error control and explicit force control. 